\title{
Intake of n-3 LCPUFA and trans-fatty acids is unrelated to development in body mass index and body fat among children
}

\author{
Xuan Ren ${ }^{1 *}$, Sofus Christian Larsen ${ }^{1}$, Lotte Lauritzen², Jeanett Friis Rohde ${ }^{1}$, Lars Bo Andersen ${ }^{3}$, Anna Bugge ${ }^{4}$, \\ Britt Wang Jensen ${ }^{5}$, Ina Olmer Specht ${ }^{1}$ and Berit Lilienthal Heitmann ${ }^{1,6,7}$
}

\begin{abstract}
Background: The number of children and adolescents with obesity has increased worldwide. Some studies have found an increase in the intake of n-3 long-chain polyunsaturated fatty acid (LCPUFA) to be beneficial for weight and obesity status. The objectives of this study were to examine if intake of trans-fatty acids (TFA) and n-3 LCPUFA at school start was associated with weight and body fat development in the following 3 and 7 years, and if substituting other fats for n-3 LCPUFA in regression models influenced weight and body fat development.

Methods: A total of 285 children (boys:130, girls:155) were included in this study. Weight, height and skinfold thickness (SF) of children were measured at age 6, 9 and 13 years by trained research personnel. Multivariate linear regression models were used to investigate the associations between n-3 LCPUFA or TFA intake and subsequent changes in body mass index (BMI) or SF. To investigate substitution effects, we constructed regression models including information on n-3 LCPUFA and all other energy given components of the diet, except for the nutrient to be substituted (all other fats and specific subgroups; saturated fatty acids (SFAs), monounsaturated fatty acids (MUFAs) and other polyunsaturated fatty acids (PUFAs)).
\end{abstract}

Results: No significant associations were observed between intake of TFA or n-3 LCPUFA and changes in BMI and SF. Also, results from regression analysis showed substituting other fats for n-3 LCPUFA did not associate with BMI or SF development.

Conclusion: The lack of associations between n-3 LCPUFA and TFA and adiposity suggests that fat composition in the diet does not play a major role in obesity development among school-aged children.

Keyword: fatty acids, children, BMl, body fat, substitution

\section{Introduction}

In the past decades, the number of children and adolescents with obesity has increased worldwide [1]. Obesity was previously considered a problem only in developed countries, but now it is also on the rise in developing

\footnotetext{
*Correspondence:

${ }^{1}$ Research Unit for Dietary Studies at The Parker Institute, Bispebjerg and Frederiksberg Hospital, Frederiksberg, Denmark

Full list of author information is available at the end of the article
}

countries [2]. Globally, over 340 million children (around $18 \%$ of children) and adolescents between 5-19 are overweight or obese [2]. Several studies suggest that children with obesity in early childhood are more prone to overweight or obesity as teenagers and adults [3, 4]. Furthermore, obesity in childhood is a significant risk factor for several lifestyle diseases [5] and premature death [6]. Therefore, it is essential to identify strategies to prevent childhood obesity [7]. 
Fat is an important nutrient during childhood, and several studies have examined the effect of different types of fatty acids intake on weight development, but the results have generally been inconsistent [8-13]. Cross-sectional studies have found an association between overweight or obesity and the intake of trans fatty acid (TFA) [12], however a previous study among 5-12 years old children found no association between serum TFAs used as biomarkers of TFA intake, and subsequent 30 months weight gain [13]. Moreover, studies in children have found that a high intake of n-3 long chain polyunsaturated fatty acid ( $n-3$ LCPUFA) or a higher ratio of PUFAs to SFA was related to less visceral obesity and more lean mass as well as a reduced risk of obesity $[8,9,14]$. In contrast, one systematic review demonstrated no association between n-3 LCPUFA and weight development [15]. This inconsistent result may result from a change in fatty acid composition when n-3 LCPUFA intake increases. In this regard a few studies among adults have found beneficial effects of replacing saturated fatty acid (SFA) with monounsaturated fatty acid (MUFA) or polyunsaturated fatty acid (PUFA) on development in body weight and obesity $[16,17]$.

There is a growing focus on modifying foods in order to replace unhealthy nutrients, e.g. replacing SFA with PUFA, as a strategy to reduce the risk of lifestyle diseases [18]. A previous study has demonstrated that replacing SFA and TFA with PUFA by different kinds of biscuits (low-fat biscuits, butter shortbread biscuits and canola shortbread biscuits) contributed to a significant weight loss and improved serum lipid in adults [19]. However, there is a general lack of studies, which examine the longterm effects of substituting the intake of specific types of fats for subsequent changes in adiposity, especially studies that investigate whether fatty acid replacement may be beneficial in the prevention of weight gain and adiposity development during childhood. Therefore, the present study aimed to investigate associations between the intake of TFA and n-3 LCPUFA among healthy 6-year old children and changes in body mass index (BMI) and skinfold thickness (SF) over the subsequent 3 and 7 years. We further examined associations with statistical substitutions of dietary intake from SFA, MUFA, and other PUFAs for n-3 LCPUFA and if the results are affected by changes in height and puberty stage.

\section{Subjects and methods \\ Subjects}

The data were derived from the Copenhagen School Child Intervention Study (CoSCIS) [20, 21]. In brief, the CoSCIS intervention delivered two additional physical activity exercise lessons per week for the first 3 years of school; improvement of schoolyard environment; selling healthy diet and snacks in school canteens; involvement of parents and health education. Children from 10 schools (27 classes) in the Ballerup municipality where enrolled in the intervention and children from 8 schools (19 classes) in the Tårnby municipality were chosen as controls [22]. In total, 1024 children were invited to participate and $68.46 \%$ (intervention: $\mathrm{n}=411$, comparison: $\mathrm{n}=290$ ) of the children and their parents or caregivers gave written informed consent. Of these, 285 (41\%) and 221 (32\%) children from both intervention and comparison groups with information on diet at age 6 years (baseline) were followed up at age 9 and 13 years, respectively, for development in BMI and skinfold thickness (Fig. 1). No main effects of the intervention were observed on physical activity, BMI or intake of macronutrients among the children after the 3-year intervention period [21, 23]. Thus, in this study we investigated the children as a cohort, taking the intervention status into account in the analyses.

\section{Dietary assessment}

Information on the children's dietary intake at both school entry (age 6 years) and grade 3 (age 9 years), was recorded for 7 consecutive days by parents or caregivers. Pre-printed food records were used to collect information on dietary habits [23], and were delivered via the teachers in closed envelopes, which the child brought home [24]. The National Food Institute, Technical University of Denmark developed and slightly modified (e.g. remove all alcoholic beverage) the food records to fit children [25]. According to the typical Danish food pattern the food records included four parts: breakfast; lunch; dinner; and in between snacks. Each meal was divided into subsections with beverage, bread, cereal, vegetables etc. and pre-printed with common foods and drinks and supplemented with an open-ended category [26]. The amount of foods consumed was assessed via household measurement scales and with photo series [24], illustrating portion sizes of common Danish foods in four or six different quantities [24] .

The completed dietary records were scanned by Eyes \& Hands version 5.2. Consumptions of nutrients were calculated using the General Intake Estimation System (version 1.000, released 26 February 2010, developed by the National Food Institute, Technical University of Denmark) and the Danish Food Consumption Databank (version 6) [24]. The dietary information included in the present study were total energy intake (MJ), total fat (energy percentage (E\%), g/d), SFA (E\%, g/d), MUFA (E\%, g/d), non-n-3 LCPUFA (E\%, g/d), n-3 LCPUFA (E\%, g/d), TFA $(\mathrm{E} \%, \mathrm{~g} / \mathrm{d})$, protein $(\mathrm{E} \%, \mathrm{~g} / \mathrm{d})$ and carbohydrate $(\mathrm{E} \%$, $\mathrm{g} / \mathrm{d}$ ) from children aged 6 years only. 


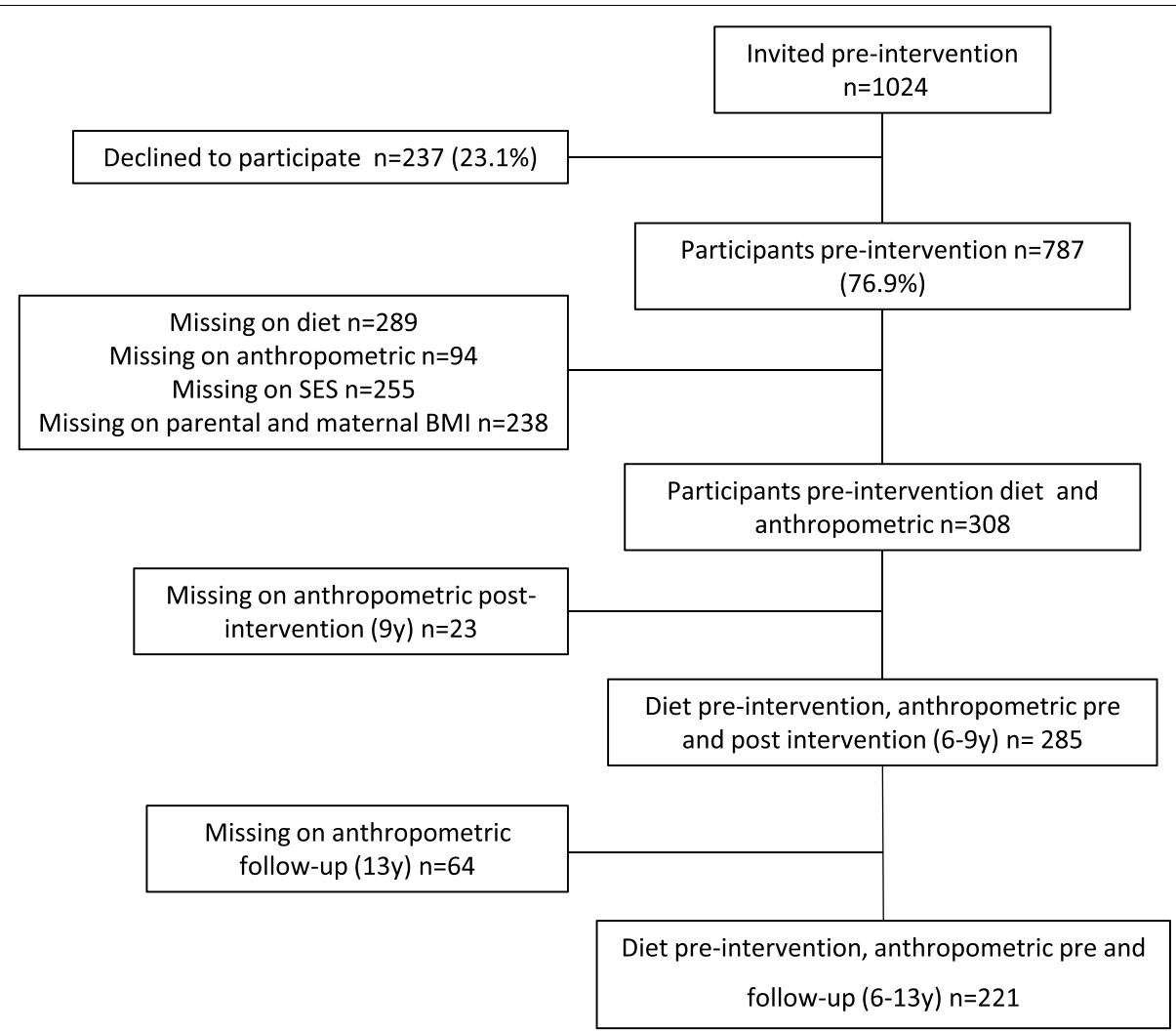

Fig. 1 Flow chart of the study population

\section{Anthropometry and other measurements}

Weight, height and skinfold thickness of children were measured at age approximately 6-, 9- and 13-years during school hours by trained researchers. Height was measured to the nearest $1 \mathrm{~mm}$ by a Harpenden stadiometer. Body weight was measured to the nearest $0.1 \mathrm{~kg}$ by a SECA electronic scale (Seca 882 , Medical Scales, NY, USA). Bicipital, tricipital, subscapular and suprailiac skinfolds were measured to the nearest $1 \mathrm{~mm}$ by a Harpenden skinfold calliper. The sum of four skinfolds ( $\sum$ SF) was calculated by summarizing the four skinfold measurements [25]. Child BMI was calculated from weight in kilograms divided by height measured in meters squared [25]. Change in BMI and skinfold thickness $(\triangle \mathrm{BMI} / \mathrm{SF})$ between different ages was calculated by subtracting BMI or $\sum$ SF at age 6 years from BMI or $\sum$ SF at age 9 or 13 years.

The assessment of pubertal stages at age 9 and 13 years was identified using the picture scale by Tanner [22]. Pubertal development in girls was assessed based on breast development (b1 indicates undeveloped breasts and b5 indicates full/completed breast development) and pubic hair growth (ph1 indicates no pubic hair yet and ph5 indicates full pubic hair growth). The assessment in boys was based on genital development (g1 indicates that genital growth has not yet begun and g5 indicates that genital development is fully develop) and pubic hair growth (same as for girls). The children were asked to evaluate their stage of sexual maturity (with same-sex lab technicians) by comparison with Tanner's photos and drawings [22].

\section{Additional information}

Physical activity was measured by the MTI 7164 activity monitor (Manufactory Technology Inc., Fort Walton Beach, FL, USA) [22] for four consecutive days (2 weekendays +2 weekdays) at age 6 years. The children were required to always wear the accelerometer, except during sleep and water-based activities. Zero counts (no activity) for 10 minutes or more were interpreted as "accelerometer not worn" and removed from the data [25]. Hence, 658 children that accumulated more than 8 hours of activity per day for at least 3 days were included in the analyses $[25,27]$.

Information on paternal and maternal weight and height was obtained by questionnaire at enrolment. Socioeconomic status (SES) categories were based on maternal education, as previous studies suggest closer 
relationship to children's dietary habits compared to paternal education or income [28-30]. Information about maternal education level was obtained using two questions as previously described [26] and the level was divided into three categories:(1) short: completed elementary school ( $\leq 10$ years) (2); medium: completed high school (12 years) or a short education (3 years) (3); long: completed college or university.

\section{Statistical analysis}

Characteristics of the study participants were presented as medians and corresponding interquartile ranges (IQR) for continuous variables and as percentages for categorical variables. All statistical analyses were performed using SAS 9.4. Statistical significance was declared if a two-sampled p-value was $<0.05$.

Linear regression models were used to investigate the association between intake of n-3 LCPUFA/TFA at age 6 years and changes in BMI or SF from age 6 to 9 years and age 6 to 13 years. We explored both the overall associations and substitution effects focusing on all other fats or specific subgroups of fat (SFAs, MUFAs and other PUFAs). To investigate substitution effects, we constructed regression models that included information on n-3 LCPUFA and all other energy given components of the diet, except for the nutrient to be substituted. The estimated corresponding regression coefficient would usually be interpreted as the effect of 1-unit n-3 LCPUFA replacing the omitted nutrient because all nutrients included in the model can be assumed constant. However, since we are using a cross-sectional measure of dietary intake, a more arcuate interpretation of the regression coefficient is the estimated difference between individuals in the dependent variable for a 1-unit higher intake of n-3 LCPUFA and a concomitantly lower intake of the omitted nutrient [31]. The substitution models were conducted defining the dietary intake as both absolute intake (g/day) and relative intake [energy percentages $(\mathrm{E} \%)]$.

All results were presented using a three-step adjustment scheme. The crude model investigated the effect of n-3 LCPUFA/TFA intake or substituting other fats for n-3 LCPUFA on changes in BMI or SF, and adjusted for sex, age and the baseline BMI or SF. For the adjusted model, we further added information on baseline physical activity, intervention/comparison group, maternal and paternal BMI and maternal education. Finally, in a third model, we added information on total energy intake. Analyses both with and without adjustment for total energy intake were conducted to explore whether potential associations were mediated by or independent of total energy intake.
As sensitivity analysis we examined if children included in our analyses differed from the remaining children (those excluded due to missing information) participating in CoSCIS, differences in baseline characteristics were tested using Wilcoxon rank sum for continuous variables and Chi-squared test for categorical variables. Sensitivity analyses were made, including additional adjustment for pubertal stage, and for the associations between fatty acids intake and BMI also for changes over time in height.

\section{Result}

In total, 285 children had complete data at follow-up after 3 years (aged 9 years) and 221 children had complete data at follow up after 7 years (aged 13 years) and were included in the analyses (Table 1). The majority of children had mothers with medium level education (completed high school); $\mathrm{n}=103,(36.1 \%)$ or long education $(\mathrm{n}=107,37.5 \%)$, and $26.3 \%$ had mothers with short education.

Their total fat intake at baseline was $78.4 \mathrm{~g} / \mathrm{d}$ (IQR: 65.1 to 93.3 ), which corresponds to $36.0 \mathrm{E} \%$ (32.5 to $38.9 \%$ ) and the intake of n-3 LCPUFA was $0.5 \mathrm{~g} / \mathrm{d}$ (0.37 to 0.64 ), corresponding to $0.2 \mathrm{E} \%$ (0.2 to 0.3$)$. The children's BMI and SF at baseline were $15.7 \mathrm{~kg} / \mathrm{m}^{2}$ (15.0 to 16.7) and 24.4 $\mathrm{mm}$ (20.8 to 28.9$)$, respectively.

The included children differed from those who were not included with respect to gender and maternal education, e.g. there was a slightly higher percentage of girls $(p=0.02)$ and longer maternal education among the included children $(\mathrm{p}=0.006)$. Apart from that there were no other overall differences at baseline between the children included and those not included in the analyses (Table S1).

Results from the regression analyses showed no significant associations in the crude analyses between intake of n-3 LCPUFA or TFA and $\triangle$ BMI over the following 3 and 7 years in models where intake was expressed as absolute intake or relative intake (Table 2). Further adjustment for baseline physical activity, intervention group, maternal and parental BMI and maternal education level gave essentially similar results, as did results after additional adjustment for energy intake (Table 2).

Furthermore, we observed no significant associations for $\triangle B M I$ over 3 and 7 years, when all other fats, SFA, MUFA or PUFA were substituted for n-3 LCPUFA, neither before or after adjusting for covariates or with the additional adjustment for total energy intake (Table 2). We further adjusted for puberty stage and $\Delta$ height in these analyses as BMI during childhood is associated with height development [32]. We observed no significant associations for any of these models (Table S3). 
Table 1 Characteristics of the participants at baseline and the follow-up examination

\begin{tabular}{|c|c|c|c|}
\hline Characteristics & Median & Q1 & Q3 \\
\hline \multicolumn{4}{|c|}{ Parental characteristics at baseline $(n=285)$} \\
\hline Paternal BMI (kg/m2) & 25.1 & 23.2 & 26.9 \\
\hline Maternal BMI (kg/m2) & 22.8 & 20.8 & 25.2 \\
\hline \multicolumn{4}{|l|}{ Maternal education $(n=285)$} \\
\hline Short n (\%) & $75(26.3 \%)$ & & \\
\hline Medium n (\%) & $103(36.1 \%)$ & & \\
\hline Long n (\%) & $107(37.5 \%)$ & & \\
\hline \multicolumn{4}{|c|}{ Characteristics at baseline (6-years of age, $n=285$ ) } \\
\hline \multicolumn{4}{|l|}{ Sex } \\
\hline Boys (\%) & $130(45.6 \%)$ & & \\
\hline Girls (\%) & $155(54.4 \%)$ & & \\
\hline Weight (kg) & 23.9 & 21.9 & 26.0 \\
\hline Height (cm) & 123.0 & 119.8 & 125.5 \\
\hline Sum of skinfold $(\mathrm{mm})^{\mathrm{a}}$ & 24.4 & 20.8 & 28.9 \\
\hline BMI $\left(\mathrm{kg} / \mathrm{m}^{2}\right)$ & 15.7 & 15.0 & 16.7 \\
\hline Physical activity score (cpm) & 695.6 & 590.5 & 837.8 \\
\hline Energy intake (MJ/d) & 8.2 & 7.2 & 9.2 \\
\hline Total fat (E\%) & 36.0 & 32.6 & 38.9 \\
\hline Total fat (g/d) & 78.4 & 65.5 & 92.9 \\
\hline SFA (E\%) & 11.8 & 10.6 & 12.9 \\
\hline SFA (g/d) & 26.1 & 21.2 & 31.0 \\
\hline MUFA (E\%) & 15.5 & 13.8 & 17.2 \\
\hline MUFA (g/d) & 33.6 & 27.8 & 40.5 \\
\hline PUFA (E\%) & 5.0 & 4.4 & 5.5 \\
\hline PUFA (g/d) & 10.9 & 9.3 & 13.4 \\
\hline n-3 LCPUFA (E\%) & 0.2 & 0.2 & 0.3 \\
\hline n-3 LCPUFA (g/d) & 0.5 & 0.4 & 0.7 \\
\hline TFA (E\%) & 0.7 & 0.5 & 0.8 \\
\hline TFA (g/d) & 1.5 & 1.1 & 1.9 \\
\hline Protein (E\%) & 14.0 & 12.8 & 15.2 \\
\hline Protein (g/d) & 66.5 & 60.0 & 77.0 \\
\hline Carbohydrate (E\%) & 48.6 & 45.7 & 51.5 \\
\hline Carbohydrate (g/d) & 253.2 & 222.3 & 285.8 \\
\hline \multicolumn{4}{|c|}{ Characteristics at follow-up at 9-year-of age $(n=285)$} \\
\hline Weight (kg) & 32.4 & 29.5 & 36.3 \\
\hline Height (cm) & 139.0 & 135.4 & 142.8 \\
\hline Sum of skinfold (mm) & 27.3 & 22.3 & 38.6 \\
\hline $\mathrm{BMI}\left(\mathrm{kg} / \mathrm{m}^{2}\right)$ & 16.9 & 15.7 & 18.2 \\
\hline \multicolumn{4}{|c|}{ Characteristics at second follow up at 13-years-of age $(n=221)$} \\
\hline Weight (kg) & 50.4 & 45.2 & 55.5 \\
\hline Height (cm) & 163.4 & 158.5 & 168 \\
\hline Sum of skinfold $(\mathrm{mm})^{\mathrm{b}}$ & 29.4 & 23.4 & 40.9 \\
\hline $\mathrm{BMI}\left(\mathrm{kg} / \mathrm{m}^{2}\right)$ & 18.7 & 17.3 & 20.4 \\
\hline
\end{tabular}

${ }^{a} n=283 ;{ }^{b} n=220$

Similarly, no associations were observed between intake of n-3 LCPUFA or TFA and $\triangle$ SF over 3 or 7 years in analysis with relative or absolute intake (Table 3 ).
Finally, we further adjusted for pubertal stage in the analyses, as skinfold thickness increase substantially when children start puberty [33]. For both TFA and n-3 LCPUFA, the results from these analyses were essentially similar to the results from other models (Table S4).

\section{Discussion}

We generally found no associations between intakes of n-3 LCPUFA or TFA at age 6 years and subsequent 3- or 7-years $\triangle \mathrm{BMI}$ and SF. Moreover, statistical substitution of other fat or SFA, MUFA, or other PUFAs with n-3 LCPUFA, showed no associations with the development in BMI or SF.

To the best of our knowledge, this study is the first to examine prospective associations between n-3 LCPUFA with and without specified replacements and adiposity development among healthy children, so we cannot compare the observed results of lack of association with results from other similar studies on children. We identified one previous randomized weight development trial giving n-3 LCPUFA supplements to children, and this study found no effect [34]. Other trial results in children generally focused on weight loss among children with overweight, and found inconsistent results [35-37].

Our results in relation to TFA are in line with the results from a previously published prospective study among 668 low- and middle-income children aged 5 to 12 years that did also not observe an association between serum TFAs and subsequent weight gain [13]. Similarly, a previous case-control study found no differences in consumption of TFA between children with a normal weight or obesity [38]. Most previous studies were conducted among adults, and a systematic review concluded that among adults a high TFA intake may lead to a small extra weight gain, albeit based on few studies that were all conducted among US adults [39].

Our study has several strengths. First, the anthropometric measurements were taken by trained researchers rather than being assessed by parents and then selfreported. Second, repeated skinfold thickness measurements over 7 years are rare and are direct measures of subcutaneous fat. Third, our multivariate linear models were adjusted for a variety of confounders as well as included energy adjusted models. Finally, substitution analyses allowed us to investigate the impact of substituting energy from fats for $\mathrm{n}-3$ LCPUFA.

The results are limited by the self-recorded diet registration with 7-day records, as this method, although generally considered among the best for diet assessment, may have some limitations for the collection of information about foods that are eaten with low frequency such as fish or seafood, which are the main sources of $n-3$ LCPUFA [40, 41]. However, the 7-day food records used 
Table 2 The association ( $\beta$ and 95\%Cl) between TFA intake and n-3 LCPUFA intake at 6 years of age and subsequent 3- and 7-year change in $\mathrm{BMI}\left(\mathrm{kg} / \mathrm{m}^{2}\right)$. Absolute intake $(\mathrm{g} / \mathrm{d})$ and relative intake models (E\%).

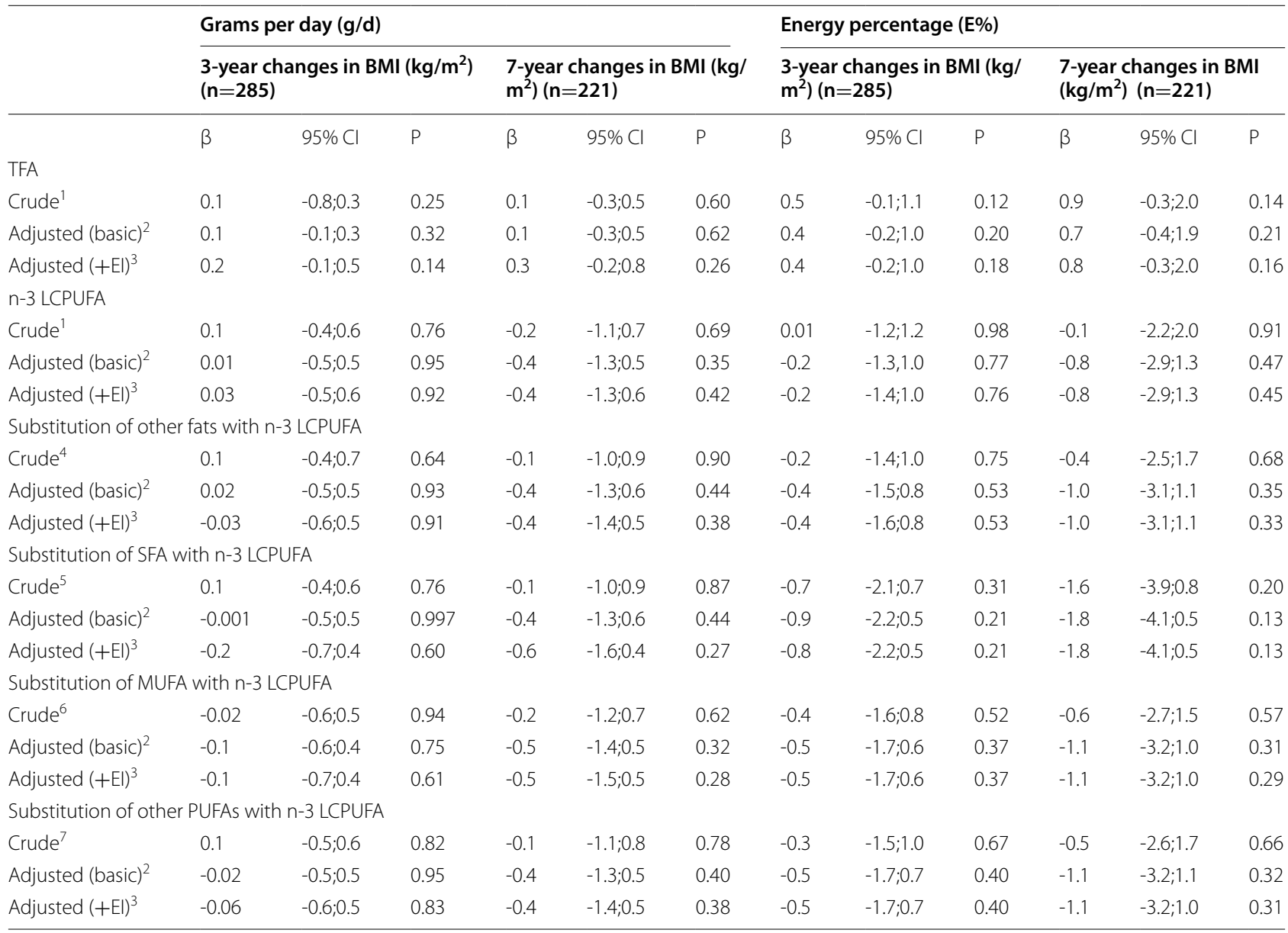

${ }^{1}$ adjusted for baseline BMI, age and sex; ${ }^{2}$ adjusted further for baseline physical activity, intervention group, maternal and parental BMI and maternal education level; ${ }^{3}$ Model adjusted further for energy intake

By holding the total energy intake, it was assumed that the increase in n-3 LCPUFA resulted in a corresponding decrease in the intake of the fat component being replaced in each model. Substitution model adjusted for baseline BMI, age, sex, baseline intake of carbohydrate and protein, baseline intake of non-specified fat

${ }^{4}$ Substitution model excluded total fat intake from the model; ${ }^{5}$ Substitution model excluded SFA intake from the model; ${ }^{6}$ Substitution model excluded MUFA intake from the model; ${ }^{7}$ Substitution model excluded PUFAs intake from the model.

in the CoSCIS study incorporated day-to-day variation across both weekdays and weekend days of one week and may therefore, be considered a suitable method to generally measure the dietary information of the children [25], although it may be a burden for the parents and require motivation and literary skills [42]. In the present study, slightly more children to better educated mothers participated, which may be a strength for data collection. However, as for other dietary instruments, the 7 -day food records can involve reporting bias and misreporting $[25,43]$. Foods with adverse health effect (e.g. SFA or TFA) are more likely to be under-reported, while those with beneficial health effects (e.g. n-3 LCPUFA) are more likely to be over-reported [44]. However, previous studies assessed the accuracy of the pre-printed record used in the present study, and the result showed only modest misreporting in children aged 7-8 years $[26,45]$. In addition, different from many other countries, most Danish children bring packed lunch boxes from home to school, so the food purchased by children at school is limited, especially at a young age [26]. Thus, the parents would have had good knowledge of their children's intake during school hours. Furthermore, another limitation might be self-reported puberty stage, as children tend to overestimate their development in the early stages of maturation and underestimate it in the later stages [46]. However, in the analyses models we also adjusted for changes in height and baseline BMI or SF. 
Table 3 The association ( $\beta$ and 95\%Cl) between TFA intake and n-3 LCPUFA intake at 6 years of age and subsequent 3- and 7-year change in SF (mm) in relative intake models (E\%).

\begin{tabular}{|c|c|c|c|c|c|c|c|c|c|c|c|c|}
\hline & \multicolumn{6}{|c|}{ Grams per day (g/d) } & \multicolumn{6}{|c|}{ Energy percentage (E\%) } \\
\hline & \multicolumn{3}{|c|}{$\begin{array}{l}\text { 3-year changes in SF ( } \mathrm{mm}) \\
(\mathrm{n}=283)\end{array}$} & \multicolumn{3}{|c|}{$\begin{array}{l}\text { 7-year changes in SF (mm) } \\
(n=219)\end{array}$} & \multicolumn{3}{|c|}{$\begin{array}{l}\text { 3-year changes in SF ( } \mathrm{mm}) \\
(\mathrm{n}=283)\end{array}$} & \multicolumn{3}{|c|}{$\begin{array}{l}\text { 7-year changes in SF } \\
(\mathrm{mm})(\mathrm{n}=219)\end{array}$} \\
\hline & $\beta$ & $95 \% \mathrm{Cl}$ & $P$ & $\beta$ & $95 \% \mathrm{Cl}$ & $P$ & $\beta$ & $95 \% \mathrm{Cl}$ & $P$ & $\beta$ & $95 \% \mathrm{Cl}$ & $P$ \\
\hline \multicolumn{13}{|l|}{ TFA } \\
\hline Crude $^{1}$ & 0.1 & $-1.3 ; 1.6$ & 0.85 & -0.01 & $-2.9 ; 2.9$ & 0.99 & 1.2 & $-3.3 ; 5.6$ & 0.61 & 3.7 & $-5.0 ; 12.4$ & 0.40 \\
\hline Adjusted (basic) ${ }^{2}$ & 0.1 & $-1.4 ; 1.5$ & 0.90 & 0.1 & $-2.8 ; 3.0$ & 0.95 & 0.7 & $-3.7 ; 5.0$ & 0.77 & 3.5 & $-5.2 ; 12.2$ & 0.43 \\
\hline Adjusted $(+\mathrm{El})^{3}$ & 0.5 & $-1.4 ; 2.5$ & 0.59 & 1.2 & $-2.7 ; 5.1$ & 0.54 & 1.0 & $-3.6 ; 5.5$ & 0.68 & 4.3 & $-4.6 ; 13.3$ & 0.34 \\
\hline \multicolumn{13}{|l|}{ n-3 LCPUFA } \\
\hline Crude $^{1}$ & 2.0 & $-1.7 ; 5.7$ & 0.28 & -0.4 & $-7.0 ; 6.2$ & 0.91 & 4.2 & $-4.6 ; 13.0$ & 0.35 & 1.6 & $-14.1 ; 17.3$ & 0.84 \\
\hline Adjusted (basic) ${ }^{2}$ & 1.6 & $-2.0 ; 5.2$ & 0.38 & -2.2 & $-8.7 ; 4.4$ & 0.52 & 2.9 & $-5.7 ; 11.6$ & 0.50 & -3.1 & $-18.8 ; 12.3$ & 0.70 \\
\hline Adjusted $(+\mathrm{El})^{3}$ & 2.2 & $-1.7 ; 6.1$ & 0.27 & -1.7 & $-8.7 ; 5.4$ & 0.64 & 2.9 & $-5.8 ; 11.6$ & 0.51 & -3.3 & $-19.0 ; 12.4$ & 0.68 \\
\hline \multicolumn{13}{|c|}{ Substitution of other fats with n-3 LCPUFA } \\
\hline Crude $^{4}$ & 2.3 & $-1.6 ; 6.3$ & 0.24 & 1.1 & $-6.0 ; 8.2$ & 0.77 & 3.0 & $-5.9 ; 11.9$ & 0.50 & 1.0 & $-14.9 ; 16.8$ & 0.90 \\
\hline Adjusted (basic) ${ }^{2}$ & 1.7 & $-2.2 ; 5.6$ & 0.39 & -1.2 & $-8.1 ; 6.0$ & 0.75 & 1.6 & $-7.1 ; 10.4$ & 0.72 & -3.2 & $-19.0 ; 13.7$ & 0.69 \\
\hline Adjusted $(+\mathrm{El})^{3}$ & 1.7 & $-2.2 ; 5.6$ & 0.40 & -1.4 & $-8.6 ; 5.7$ & 0.69 & 1.6 & $-7.1 ; 10.4$ & 0.72 & -3.4 & $-19.3 ; 12.4$ & 0.67 \\
\hline \multicolumn{13}{|c|}{ Substitution of SFA with n-3 LCPUFA } \\
\hline Crude $^{5}$ & 2.3 & $-1.6 ; 6.3$ & 0.24 & 1.0 & $-6.1 ; 8.1$ & 0.78 & -2.3 & $-12.4 ; 7.8$ & 0.66 & -11.2 & $-29.0 ; 6.6$ & 0.21 \\
\hline Adjusted (basic) ${ }^{2}$ & 1.7 & $-2.2 ; 5.6$ & 0.39 & -1.2 & $-8.3 ; 6.0$ & 0.75 & -3.1 & $-12.9 ; 6.8$ & 0.54 & -12.5 & $-30.2 ; 5.2$ & 0.16 \\
\hline Adjusted $(+\mathrm{El})^{3}$ & 0.7 & $-3.5 ; 5.0$ & 0.74 & -3.6 & $-11.3 ; 4.1$ & 0.36 & -3.1 & $-13.0 ; 6.8$ & 0.54 & -12.5 & $-30.2 ; 5.2$ & 0.17 \\
\hline \multicolumn{13}{|c|}{ Substitution of MUFA with n-3 LCPUFA } \\
\hline Crude $^{6}$ & 2.0 & $-2.0 ; 6.0$ & 0.32 & 0.2 & $-6.9 ; 7.3$ & 0.95 & 1.8 & $-7.1 ; 10.8$ & 0.69 & -0.04 & $-15.9 ; 15.9$ & 0.99 \\
\hline Adjusted (basic) ${ }^{2}$ & 1.5 & $-2.4 ; 5.4$ & 0.45 & -1.7 & $-8.8 ; 5.5$ & 0.64 & 0.8 & $-8.0 ; 9.6$ & 0.85 & -3.6 & $-19.5 ; 12.3$ & 0.66 \\
\hline Adjusted $(+ \text { El) })^{3}$ & 1.1 & $-2.8 ; 5.1$ & 0.58 & -1.8 & $-9.0 ; 5.4$ & 0.62 & 0.8 & $-8.0 ; 9.6$ & 0.86 & -3.8 & $-19.7 ; 12.1$ & 0.64 \\
\hline \multicolumn{13}{|c|}{ Substitution of other PUFAs with n-3 LCPUFA } \\
\hline Crude $^{7}$ & 2.3 & $-1.6 ; 6.3$ & 0.25 & 0.7 & $-6.4 ; 7.8$ & 0.85 & 1.8 & $-7.1 ; 10.8$ & 0.69 & -0.04 & $-15.9 ; 15.9$ & 0.99 \\
\hline Adjusted (basic) ${ }^{2}$ & 1.7 & $-2.2 ; 5.6$ & 0.39 & -1.4 & $-8.5 ; 5.7$ & 0.70 & 0.8 & $-8.0 ; 9.6$ & 0.85 & -3.6 & $-19.5 ; 12.3$ & 0.66 \\
\hline Adjusted $(+\mathrm{El})^{3}$ & 1.6 & $-2.3 ; 5.6$ & 0.42 & -1.3 & $-8.5 ; 5.9$ & 0.72 & 0.8 & $-8.0 ; 9.6$ & 0.86 & -3.8 & $-19.7 ; 12.1$ & 0.64 \\
\hline
\end{tabular}

${ }^{1}$ adjusted for baseline SF, age and sex; ${ }^{2}$ adjusted further for baseline physical activity, intervention group, maternal and parental BMI and maternal education level; ${ }^{3}$ Model adjusted further for energy intake

By holding the total energy intake, it was assumed that the increase in n-3 LCPUFA resulted in a corresponding decrease in the intake of the fat component being replaced in each model. Substitution crude model adjusted for baseline SF, age, sex, baseline intake of carbohydrate and protein, baseline intake of non-specified fat

${ }^{4}$ Substitution model excluded total fat intake from the model; ${ }^{5}$ Substitution model excluded SFA intake from the model; ${ }^{6}$ Substitution model excluded MUFA intake from the model; ${ }^{7}$ Substitution model excluded PUFAs intake from the model.

In the present study, the intake of n-3 LCPUFA defined less than 20 percent the BMI or SF variation at age 7 or 13 years. The lack of associations between intake of fats and adiposity in our study may also be due to low accuracy of the fatty acids information in relation to the quality of the fatty acid composition data in the nutritional tables [47]. Therefore, bias in the fatty acids intake estimates in the study may have attenuated potential associations between the specific fatty acid and the weight and adiposity development. Our analyses included several confounders, but some potential unmeasured confounding may still have remained, and some residual confounding is inevitable. Lack of control for those confounders may also have attenuated potential true associations [48].
The assessment of growth in weight and body fat is complicated by the age of the children in the present study. They were 6 years at baseline and thus, around the age of the adiposity rebound, where some may not have reached the turning point yet, while others have started to increase in BMI [49]. Also, after 3- and especially 7 - years follow-up, the children were in the different stages of puberty [50], which may have resulted in large variation in height due to the pubertal growth spurt [51]. Uncertainty in the outcome assessment may thus have an effect on power and on the ability to detect potential associations. However, we adjusted our results by including change in height and puberty stage and found essentially similar results, suggesting that 
difference in puberty staging and change in height did not substantially confound our findings. The relatively small sample size may, and thus low power of the study, can have increased the risk of type II error and chance findings [52].

As shown in a previous study, the CoSCIS intervention did not have an overall effect on diet intake, except for a decrease in the intake of SFA among children of mothers with long education [26]. In the present study, the mothers of the children included in the analyses tended to be more educated than did mothers of those not included. This may reduce the generalizability of the results, but also highlights the importance of considering parental education in relation to children's dietary intake and risk of obesity.

\section{Conclusion}

This longitudinal study did not provide evidence to support that a high TFA or a low n-3 LCPUFA intake among 6 year-old children was related to subsequent 3 and 7 years increase in BMI or SF. Statistically substituting other fats for n-3 LCPUFA also did not influence 3 - and 7-years weight development. Large prospective studies are needed to better understand the relationships between fatty acids intake and development of overweight and obesity during childhood.

\section{Supplementary Information}

The online version contains supplementary material available at https://doi. org/10.1186/s40795-021-00493-5.

Additional file 1.

\section{Acknowledgements}

The author would like to thank all participating children and their families, the school principals and teachers, the politicians and employees in the local authorities of Ballerup and Tårnby.

\section{Authors' contributions}

$\angle B A$ and $A B$ participated in the data collection. BLH was responsible for the collection of dietary data and designed this study. XR wrote the first draft. All authors reviewed and commented on subsequent drafts and approved the final version of the manuscript.

\section{Funding}

The CoSCIS study was funded by the Tryg Foundation and the Danish Heart Association. The Parker Institute, Bispebjerg and Frederiksberg Hospital, is supported by a core grant from the Oak Foundation (OCAY-18-774-OFIL). The funders had no role in study design, collection, analysis and interpretation of data, writing of the paper or decision to submit for publication.

\section{Availability of data and materials}

The data presented in this study are available on request to bfh-dl-eek@regio nh.dk. The data are not publicly available due to the participant's privacy and data protection.

\section{Declaration}

\section{Ethics approval and consent to participate}

The present study has been approved the Capital Region's Committee on Health Research Ethics (the former Ethical Committee of Copenhagen County; case no. KA0001 $1 \mathrm{gm}$ ) and conducted according to the guidelines laid down in the Helsinki declaration of 1975 as revised in 1983. Written information about the study was given to all school leaders, teachers and parents before the start of the study. Informed consent was obtained from the parents/legal guardian of the participants.

\section{Consent for publication}

Not applicable

\section{Competing interests}

None

\section{Author details}

${ }^{1}$ Research Unit for Dietary Studies at The Parker Institute, Bispebjerg and Frederiksberg Hospital, Frederiksberg, Denmark. ${ }^{2}$ Department of Nutrition, Exercise and Sports, Paediatric and International Nutrition, University of Copenhagen, Copenhagen, Denmark. ${ }^{3}$ Department of Education, Arts and Sport, Western Norway University of Applied Sciences, Sogndal, Norway. ${ }^{4}$ Department of Midwifery, Physiotherapy, Occupational Therapy and Psychomotor Therapy, University College Copenhagen, Copenhagen, Denmark. ${ }^{5}$ Center for Clinical Research and Prevention, Bispebjerg and Frederiksberg Hospital, Frederiksberg, Denmark. ${ }^{6}$ The Boden Group, Faculty of Medicine and Health, Sydney University, Sydney, Australia. ${ }^{7}$ Department of Public Health, Section for General Medicine, University of Copenhagen, Copenhagen, Denmark.

Received: 9 September 2021 Accepted: 16 December 2021

Published online: 03 January 2022

\section{References}

1. Sanyaolu A, Okorie C, Qi X, Locke J, Rehman S. Childhood and Adolescent Obesity in the United States: A Public Health Concern. Glob Pediatr Health. 2019;6:2333794X19891305-2333794X.

2. WHO. Obesity and overweight 2018 [Available from: https://www.who. int/en/news-room/fact-sheets/detail/obesity-and-overweight.

3. Geserick M, Vogel M, Gausche R, LipekT, Spielau U, Keller E, et al. Acceleration of BMI in Early Childhood and Risk of Sustained Obesity. The New England journal of medicine. 2018;379(14):1303-12.

4. Biro FM, Wien M. Childhood obesity and adult morbidities. The American journal of clinical nutrition. 2010:91(5):1499S-505S.

5. Sahoo K, Sahoo B, Choudhury AK, Sofi NY, Kumar R, Bhadoria AS. Childhood obesity: causes and consequences. J Family Med Prim Care. 2015;4(2):187-92.

6. Reilly JJ. Descriptive epidemiology and health consequences of childhood obesity. Best practice \& research Clinical endocrinology \& metabolism. 2005;19(3):327-41.

7. Stratakis N, Gielen M, Margetaki K, Godschalk RW, van der Wurff I, Rouschop S, et al. Polyunsaturated fatty acid levels at birth and child-toadult growth: Results from the MEFAB cohort. Prostaglandins, leukotrienes, and essential fatty acids. 2017;126:72-8.

8. Standl M, Demmelmair H, Koletzko B, Heinrich J. Cord blood n-3 LC-PUFA is associated with adiponectin concentrations at 10 years of age. Prostaglandins, leukotrienes, and essential fatty acids. 2015;96:51-5.

9. Cardel M, Lemas DJ, Jackson KH, Friedman JE, Fernández JR. Higher Intake of PUFAs Is Associated with Lower Total and Visceral Adiposity and Higher Lean Mass in a Racially Diverse Sample of Children. J Nutr. 2015; 145(9):2146-52.

10. Bender N, Portmann M, Heg Z, Hofmann K, Zwahlen M, Egger M. Fish or n3-PUFA intake and body composition: a systematic review and metaanalysis. Obesity reviews : an official journal of the International Association for the Study of Obesity. 2014;15(8):657-65.

11. Pedersen MH, Mølgaard C, Hellgren LI, Matthiessen J, Holst JJ, Lauritzen L. The Effect of Dietary Fish Oil in addition to Lifestyle Counselling on Lipid Oxidation and Body Composition in Slightly Overweight Teenage Boys. Journal of Nutrition and Metabolism. 2011;2011:348368. 
12. Scholz A, Navarrete-Muñoz EM, García-de-la-Hera M, Fernandez-Somoano A, Tardon A, Santa-Marina $L$, et al. Association between trans fatty acid intake and overweight including obesity in 4 to 5 -year-old children from the INMA study. Pediatr Obes. 2019;14(9):e12528.

13. Baylin A, Perng W, Mora-Plazas M, Marin C, Villamor E. Serum Trans Fatty Acids Are Not Associated with Weight Gain or Linear Growth in School-Age Children. J Nutr. 2015;145(9):2102-8.

14. Mak IL, Cohen TR, Vanstone CA, Weiler HA. Increased adiposity in children with obesity is associated with low red blood cell omega-3 fatty acid status and inadequate polyunsaturated fatty acid dietary intake. Pediatric Obesity. 2020;15(12):e12689.

15. Middleton P, Gomersall JC, Gould JF, Shepherd E, Olsen SF, Makrides M. Omega-3 fatty acid addition during pregnancy. Cochrane Database of Systematic Reviews. 2018. https://doi.org/10.1002/14651858.CD003402. pub3(11)

16. Beulen Y, Martínez-González MA, van de Rest O, Salas-Salvadó J, Sorlí JV, Gómez-Gracia E, et al. Quality of Dietary Fat Intake and Body Weight and Obesity in a Mediterranean Population: Secondary Analyses within the PREDIMED Trial. Nutrients. 2018;10(12)

17. Liu X, Li Y, Tobias DK, Wang DD, Manson JE, Willett WC, et al. Changes in Types of Dietary Fats Influence Long-term Weight Change in US Women and Men. J Nutr. 2018;148(11):1821-9.

18. Lenighan YM, McNulty BA, Roche HM. Dietary fat composition: replacement of saturated fatty acids with PUFA as a public health strategy, with an emphasis on a-linolenic acid. Proceedings of the Nutrition Society. 2019;78(2):234-45

19. Noakes M, Clifton PM. Changes in plasma lipids and other cardiovascular risk factors during 3 energy-restricted diets differing in total fat and fatty acid composition. Am J Clin Nutr. 2000;71(3):706-12.

20. Andersen LBF, Karsten. Sundhedsmæssige aspekter af fysisk aktivitet hos børn. 2006.

21. Bugge A, El-Naaman B, Dencker M, Froberg K, Holme IM, McMurray RG, et al. Effects of a three-year intervention: the Copenhagen School Child Intervention Study. Medicine and science in sports and exercise. 2012;44(7):1310-7.

22. Hasselstrøm HA, Karlsson MK, Hansen SE, Grønfeldt V, Froberg K, Andersen LB. A 3-year physical activity intervention program increases the gain in bone mineral and bone width in prepubertal girls but not boys: the prospective copenhagen school child interventions study (CoSCIS). Calcified tissue international. 2008;83(4):243-50.

23. Jensen BW, Nielsen BM, Husby I, Bugge A, El-Naaman B, Andersen LB, et al. Association between sweet drink intake and adiposity in Danish children participating in a long-term intervention study. Pediatric obesity. 2013;8(4):259-70.

24. van Vught AJ, Heitmann BL, Nieuwenhuizen AG, Veldhorst MA, Andersen $L B$, Hasselstrom $\mathrm{H}$, et al. Association between intake of dietary protein and 3-year-change in body growth among normal and overweight 6-year-old boys and girls (CoSCIS). Public Health Nutr. 2010;13(5):647-53.

25. Jensen BW. effects of a school-based intervention on dietary intake, and the association between beverage intake and the development of obesity during childhood - Results from The Copenhagen School Child Intervention Study: University of Southern Denmark; 2013.

26. Jensen BW, von Kappelgaard LM, Nielsen BM, Husby I, Bugge A, El-Naaman $B$, et al. Intervention effects on dietary intake among children by maternal education level: results of the Copenhagen School Child Intervention Study (CoSCIS). British Journal of Nutrition. 2015;113(6):963-74.

27. Eiberg S, Hasselstrom H, Grønfeldt V, Froberg K, Svensson J, Andersen LB. Maximum oxygen uptake and objectively measured physical activity in Danish children 6-7 years of age: the Copenhagen school child intervention study. British Journal of Sports Medicine. 2005;39(10):725.

28. Moreira P, Santos S, Padrão P, Cordeiro T, Bessa M, Valente H, et al. Food patterns according to sociodemographics, physical activity, sleeping and obesity in Portuguese children. Int J Environ Res Public Health. 2010;7(3):1121-38

29. Northstone $K$, Emmett $P$, The AST. Multivariate analysis of diet in children at four and seven years of age and associations with socio-demographic characteristics. European Journal of Clinical Nutrition. 2005;59(6):751-60.

30. Cribb VL, Jones LR, Rogers IS, Ness AR, Emmett PM. Is maternal education level associated with diet in 10-year-old children? Public Health Nutrition. 2011;14(11):2037-48.

31. Song M, Giovannucci E. Substitution analysis in nutritional epidemiology: proceed with caution. Eur J Epidemiol. 2018;33(2):137-40.
32. He Q, Karlberg J. BMI in Childhood and Its Association with Height Gain, Timing of Puberty, and Final Height. Pediatric Research. 2001;49(2):244-51.

33. Vizmanos B, Martí-Henneberg C. Puberty begins with a characteristic subcutaneous body fat mass in each sex. European Journal of Clinical Nutrition. 2000:54(3):203-8.

34. Svensson V, Johansson E, Fischer M, Deng SL, Hagströmer M, Danielsson P. Omega-3 fatty acids does not affect physical activity and body weight in primary school children - a double-blind randomized placebo-controlled trial. Scientific Reports. 2018:8(1):12725

35. Vasickova L, Stavek P, Suchanek P. Possible effect of DHA intake on body weight reduction and lipid metabolism in obese children. Neuro endocrinology letters. 2011;32(Suppl 2):64-7.

36. See VHL, Mori TA, Prescott SL, Beilin L, Burrows S, Huang R-C. Cardiometabolic Risk Factors at 5 Years After Omega-3 Fatty Acid Supplementation in Infancy. Pediatrics. 2018;142(1):e20162623.

37. López-Alarcón M, Inda-Icaza P, Márquez-Maldonado MC, Armenta-Álvarez A, Barbosa-Cortés L, Maldonado-Hernández J, et al. A randomized control trial of the impact of LCPUFA- $\omega 3$ supplementation on body weight and insulin resistance in pubertal children with obesity. Pediatric obesity. 2019:14(5):e12499.

38. Larqué E, Gil-Campos M, Ramírez-Tortosa MC, Linde J, Cañete R, Gil A. Postprandial response of trans fatty acids in prepubertal obese children. International Journal of Obesity. 2006;30(10):1488-93.

39. Thompson A, Minihane A, Williams C. Trans fatty acids and weight gain. International journal of obesity. 2005;2011(35):315-24.

40. Herter-Aeberli I, Graf C, Vollenweider A, Häberling I, Srikanthan P, Hersberger $M$, et al. Validation of a Food Frequency Questionnaire to Assess Intake of n-3 Polyunsaturated Fatty Acids in Switzerland. Nutrients. 2019;11(8):1863.

41. Damsgaard CT, Lauritzen L, Hauger H, Vuholm S, Teisen MN, Ritz C, et al. Effects of oily fish intake on cardiovascular risk markers, cognitive function, and behavior in school-aged children: study protocol for a randomized controlled trial. Trials. 2016;17(1):510.

42. Willett WC. Nutritional Epidemiology: Oxford University Press, USA; 1998

43. Høidrup S, Andreasen AH, Osler M, Pedersen AN, Jørgensen LM, Jørgensen T, et al. Assessment of habitual energy and macronutrient intake in adults: comparison of a seven day food record with a dietary history interview. European journal of clinical nutrition. 2002;56(2):105-13.

44. Macdiarmid J, Blundell J. Assessing dietary intake: Who, what and why of under-reporting. Nutr Res Rev. 1999;11:231-53.

45. Rothausen BW, Matthiessen J, Groth MV, Brockhoff PB, Andersen LF, Trolle E. Comparison of estimated energy intake from $2 \times 24$-hour recalls and a seven-day food record with objective measurements of energy expenditure in children. Food Nutr Res. 2012;56:10.3402/fnr.v56i0.12221.

46. Schlossberger NM, Turner RA, Irwin CE Jr. Validity of self-report of pubertal maturation in early adolescents. J Adolesc Health. 1992;13(2):109-13.

47. Fabiansson S. Precision in nutritional information declarations on food labels in Australia. Asia Pacific journal of clinical nutrition. 2006;15:451-8.

48. Skelly AC, Dettori JR, Brodt ED. Assessing bias: the importance of considering confounding. Evid Based Spine Care J. 2012;3(1):9-12.

49. Rolland-Cachera MF, Deheeger M, Maillot M, Bellisle F. Early adiposity rebound: causes and consequences for obesity in children and adults. International journal of obesity. 2005;2006(30 Suppl 4):S11-7.

50. Parent A-S, Teilmann G, Juul A, Skakkebaek NE, Toppari J, Bourguignon J-P. The Timing of Normal Puberty and the Age Limits of Sexual Precocity: Variations around the World, Secular Trends, and Changes after Migration. Endocrine Reviews. 2003;24(5):668-93.

51. Abbassi V. Growth and Normal Puberty. Pediatrics. 1998;102(Supplement 3):507.

52. Columb MO, Atkinson MS. Statistical analysis: sample size and power estimations. BJA Education. 2016:16(5):159-61.

\section{Publisher's Note}

Springer Nature remains neutral with regard to jurisdictional claims in published maps and institutional affiliations. 